\title{
Correlating Dynamometer Testing to In-Use Fleet Results of Plug-In Hybrid Electric Vehicles
}

\author{
Michael Duoba ${ }^{1}$, Richard Carlson ${ }^{2}$, Forrest Jehlik ${ }^{3}$, John Smart $^{4}$, Sera White ${ }^{5}$ \\ ${ }^{\prime}$ Argonne National Laboratory, 9700 South Cass Ave, Argonne, IL 60439,mduoba@anl.gov \\ 22Argonne National Laboratory, 9700 South Cass Ave, Argonne, IL 60439,fjehlik@anl.gov \\ ${ }^{3}$ Argonne National Laboratory, 9700 South Cass Ave, Argonne, IL 60439, rwcarlson@anl.gov \\ ${ }^{4}$ Idaho National Laboratory, 2351 N Blvd, Idaho Falls, ID, 83415, john.smart@inl.gov \\ ${ }^{5}$ Idaho National Laboratory, 2351 N Blvd, Idaho Falls, ID, 83415, sera.white@inl.gov
}

\begin{abstract}
The Untied States Department of Energy is engaged in the research, development and deployment of PHEV technology through the "Advanced Vehicle Testing Activity." In this program, data has been collected from a Prius PHEV converted by Hymotion ${ }^{\mathrm{TM}}$ using industry standard dynamometer testing procedures and from in-use operation of fleets. Differences in fuel-only consumption rates observed in the on-road data compared to the dynamometer results were fairly dramatic. Correlations of the driving style and conditions of dynamometer testing and on-road conditions were made possible by comprehensive onroad loggers. A model was developed to infer driving intensity from the on-road dataset from data load measurement during dynamometer testing. The reasons for the shortfall are detailed in the paper. They range from driver aggressiveness, accessory loads, ambient temperature (weather), and the high sensitivity the control system exhibits with increased acceleration pedal demand and higher average cruising speeds.
\end{abstract}

Keywords: PHEV (plug in hybrid electric vehicle), energy consumption, vehicle performance

\section{Introduction}

Recent advances in battery technology for vehicle electrification have potential to displace significant amounts of petroleum by using electrical grid energy. Interest in the plug-in hybrid (PHEV) has been fuelled by a cottage industry that converts conventional hybrids to PHEV operation with the addition of supplemental batteries and control system modifications. The claims of " $+100 \mathrm{MPG"} \mathrm{come}$ largely from results from standard dynamometer test results but are not necessarily taken in the context of what the average driver may experience. Comparisons of standard test results with on-road experience will address many questions about the performance of converted HEVs to PHEV operation.

The U.S. Department of Energy's Advanced Vehicle Testing Activity (AVTA) performs independent testing to assess the energy efficiency of advanced technology vehicles, including as PHEVs. AVTA is conducting a comprehensive PHEV testing and evaluation program, with testing in laboratory, track, and on-road environments. Argonne National Laboratory performs AVTA's light-duty dynamometer testing. The Idaho National Laboratory conducts on-road testing and fleet demonstrations for AVTA. 
The purpose of this paper is to correlate results from AVTA's dynamometer testing to in-use fleet vehicle performance.

\section{Dynamometer Testing}

It is current practice in the automotive industry to use standard dynamometer drive cycles during vehicle technology evaluation and benchmarking testing. Most tests occur under standard laboratory conditions of $20^{\circ} \mathrm{C}$ with no cabin heating or cooling. The dynamometer road load is set based upon closed track coast down testing during ideal conditions (negligible wind, driving perfectly straight, smooth pavement).

These conditions are a very repeatable and equitable standard for comparison purposes, but will often under-predict fuel consumption compared to actual day-to-day driving. Weather, wind and road conditions are part of the reason why the U.S. Environmental Protection Agency (U.S. EPA) adjusts the results of city and highway test results for the "label" fuel economy value [1].

\subsection{Test Cycles}

There are many standard test cycles developed over the last several decades that are used in chassis dynamometer testing. Cycles are defined as time-speed traces roughly 10 to 30 minutes in length. In 2008, new U.S. EPA labelling methods were adopted to calculate the city and highway fuel economy by including results from five separate tests that include various levels of aggressive driving, speeds, and ambient conditions. The Urban Dynamometer Driving Schedule (UDDS), Highway Fuel Economy Driving Schedule (HWFEDS or HWY), and the US06 cycle are tested under standard lab ambient temperature with no additional solar simulation. The SC03 cycle is tested at elevated temperature $\left(35^{\circ} \mathrm{C}\right)$ and with artificial solar lighting at a load of $850 \mathrm{~W} / \mathrm{m}^{3}$. There is also a $-7^{\circ} \mathrm{C}$ UDDS test.

The UDDS and HWFEDS cycles were the original certification cycles developed in the late 1960's to represent realistic drive cycles. These cycles are generally regarded as being less aggressive than the way people typically drive today. The US06 cycle was developed to find "off-cycle" high load emissions. Thus, is can be considered a limit cycle; that is, it contains speeds and acceleration rates representing extremely aggressive driving. Another cycle used in vehicle testing is the LA92, a cycle specifically designed to represent modern traffic speeds and acceleration rates.

Table 1: Standard Test Cycle Statistics

\begin{tabular}{|l|c|c|c|c|}
\cline { 2 - 5 } \multicolumn{1}{c|}{} & $\begin{array}{c}\text { Ave } \\
\text { MPH }\end{array}$ & $\begin{array}{c}\text { Ave non- } \\
\text { zero MPH }\end{array}$ & $\begin{array}{c}\text { Max } \\
\text { MPH }\end{array}$ & $\begin{array}{c}\text { Intensity } \\
\text { Wh/mi }\end{array}$ \\
\hline UDDS & 19.5 & 24.1 & 56.7 & 165 \\
\hline HWY & 47.6 & 48.6 & 59.9 & 133 \\
\hline US06 & 48.0 & 51.8 & 80.3 & 243 \\
\hline LA92 & 24.6 & 29.4 & 67.2 & 199 \\
\hline SC03 & 21.4 & 26.6 & 54.8 & 173 \\
\hline
\end{tabular}

Some metrics describing test schedules are shown in Table 1. Average non-zero speed is the average of vehicle speed greater than zero in a cycle. This eliminates the effect of vehicle idle time on average speed. The parameter "Intensity" is the positive propulsion energy per mile needed to overcome the road losses and inertia of each test cycle, measured in the $\mathrm{Wh} / \mathrm{km}$ (and $\mathrm{Wh} / \mathrm{mi}$ ). More on this parameter is explained in section 3.4.1.

Intensity numbers shown in Table 1 are specific to the Toyota Prius with Hymotion PHEV conversion module from A123Systems. This vehicle, also referred to as the Hymotion Prius, contains a supplemental $5 \mathrm{kWh}$ lithium ion battery pack, which is charged from the electrical grid.

In general, the standard dynamometer test procedures typically run the aforementioned drive schedules twice. The HWY, US06, and SC03 tests are run with the first drive schedule unsampled as a warm-up cycle. The UDDS, LA92 and $-7^{\circ} \mathrm{C}$ UDDS tests are run without a warm-up. These tests represent the initial start and warm-up of the beginning and a "hot start" after warm-up has occurred. The two UDDS and LA92 schedules have a 10 minute rest (key off) between cycles. For conventional vehicles and all hybrids doing a charge-sustaining UDDS test, there is a weighting ratio to represent several daily trips, some occurring warm and some (like the first start of the day) occurring cold (engine and exhaust at ambient temperature).

\subsection{PHEV Testing}

PHEVs operate in both charge-depleting (CD) and charge-sustaining (CS) modes. Developing procedures to capture all of the operating modes of a PHEV while maintaining original test conditions and assumptions of the various legacy procedures is a challenging task $[2,3]$. In short, the method used by researchers is to repeat the drive schedules 
back-to-back until a satisfactory chargebalanced cycle is achieved (at which time the test is terminated). Gasoline fuel and battery energy consumption for each test cycle is captured and parameters such as charge-depleting range are determined.

The methodology described in SAE J1711 to find the "final answer" from all results in testing is Utility Factor (UF) weighting. In-use daily driving statistics are applied to carefully weight all the tests in the charge-depleting test with those in the charge-sustaining test [4]. This methodology provides an estimation of in-use gasoline fuel economy and electrical energy consumption. The results of dynamometer testing for the Hymotion Prius are found in Table 2.

Table 2: Depleting, Sustaining, and UF-Weighted Standard Test Cycle Results for the Hymotion Prius

\begin{tabular}{|lccc|cc|ccc|}
\hline & \multicolumn{3}{c|}{ CD } & \multicolumn{2}{c|}{ CS } & \multicolumn{3}{c|}{ UF-Weighted } \\
& MPG & L/100km Wh/mi & MPG & L/100km & MPG & L/100km Wh/mi \\
\hline UDDS & 181.1 & 1.3 & 131.9 & 66.6 & 3.5 & 91.6 & 2.57 & 96.6 \\
\hline HWY & 121.0 & 1.9 & 119.5 & 63.5 & 3.7 & 85.2 & 2.76 & 91.5 \\
\hline US06 & 52.9 & 4.4 & 78.7 & 43.2 & 5.4 & 49.5 & 4.75 & 80.9 \\
\hline SC03 & 91.2 & 2.6 & 187.5 & 38.0 & 6.2 & 49.8 & 4.72 & 101.0 \\
\hline LA92 & 88.1 & 2.7 & 113.2 & 50.0 & 4.7 & 63.8 & 3.68 & 93.5 \\
\hline
\end{tabular}

An important observation is the fact that the vehicle does indeed achieve over 100 MPG in some cycles while in charge-depleting mode. The US06 has very high driving demands and the $\mathrm{SC} 03$ uses the air conditioner $(\mathrm{A} / \mathrm{C})$ during the elevated temperature test. Consequently, the respective fuel consumption results of these two cycles are much higher.

\section{On-Road Data}

The AVTA monitors PHEV in-use performance through its fleet demonstration program. In this program, the AVTA has collected in-use data from 8 different PHEV conversion models, including the Hymotion Prius. These vehicle models are represented in a fleet of 155 vehicles operated in 23 U.S. states and Canadian provinces by over 75 organizations. Vehicles are equipped with on-board data loggers, which record time history data. Over 360,000 miles have been logged since the program's onset in late 2007. While the majority of vehicles are operated in commercial fleets, approximately $10 \%$ of the miles driven to date were logged in vehicles used for private use [5].

\subsection{Summary of Hymotion Prius On- Road Results}

The most common question a person may ask about a new fuel saving technology is "What is the fuel economy?" To answer this question, fleet fuel economy was processed and a single aggregate MPG was calculated. Data from 73 Hymotion Prius vehicles with the V2Green data logger from June 1, 2008 to Feb 28, 2009 were chosen for analysis. Statistics describing these data are shown in Table 3.

Table 3: Large On-Road Data set Statistics

\begin{tabular}{|l|r|}
\hline Number of distinct cars & 73 \\
Total miles & 242628 \\
Total number of trips & 24714 \\
CD miles & 87109 \\
CS miles & 155519 \\
$\%$ CD & 0.36 \\
$\%$ CS & 0.64 \\
\hline
\end{tabular}

Table 4 shows that the total miles driven per total gallons fuel consumed is 49.1 MPG (4.79 $\mathrm{L} / 100 \mathrm{~km}$ ). Algorithms were developed to segregate trips in charge-depleting and charge sustaining operation, and operating mode-specific fuel economy results were calculated. Table 4 shows the charge-depleting fuel economy of 61.9 MPG, compared to the dynamometer CD results (from Table 2) of 53 to $181 \mathrm{MPG}$.

Table 4: Overall Fuel Consumption Results

\begin{tabular}{|l|c|c|}
\multicolumn{1}{c}{} & \multicolumn{1}{c}{ MPG } & \multicolumn{1}{c}{ L/100km } \\
\hline Overall & 49.1 & 4.79 \\
Charge Depleting (CD) & 61.9 & 3.80 \\
Charge-Sustaining (CS) & 43.3 & 5.43 \\
\hline
\end{tabular}

\subsection{Subset of 1200 On-Road Trips}

From the large fleet data set, a more manageable sample of 1200 trips was selected. Trips with distance greater than 1 mile were randomly selected for this sample. The sample subset, summarized in Table 5, and the parent set share similar results. The remainder of this paper references the 1200 trip subset, except where noted. This data set is analyzed to find reasons to explain the difference between on-road gasoline and electrical energy consumption and the results from standard dynamometer test procedures. 
Table 5: Overall Results of a Subset with 1200 Trips

\begin{tabular}{|c|cccc|cc|c|}
\cline { 2 - 7 } \multicolumn{1}{c|}{} & Total mi & Total gal & MPG & Total $\mathrm{kWh}$ & $\mathbf{w h} / \mathbf{m i}$ & \% of Dist \\
\hline CD & 5453 & 87.4 & $\mathbf{6 2 . 4}$ & -708.5 & $\mathbf{1 2 9 . 9}$ & $35 \%$ \\
CS & 10134 & 228.7 & $\mathbf{4 4 . 3}$ & 21.3 & $\mathbf{- 2 . 1}$ & $65 \%$ \\
\hline \hline Total & 15587 & 316.1 & $\mathbf{4 9 . 3}$ & -687.2 & $\mathbf{4 4 . 1}$ & \multicolumn{1}{c}{} \\
\hline
\end{tabular}

\subsection{Charging Frequency and Distance between Charging Events}

One principal assumption for processing dynamometer test results to represent "real life" is the frequency with which a vehicle owner would charge the vehicle. The current consensus among researchers is to assume one charge per day of driving [6]. This assumption is based on the premise that days when a vehicle operator does not charge - perhaps due to forgetfulness or the absence of charging infrastructure - will be offset on other days by "opportunity charging," or charging more than once during a day (at home or at locations with charging stations).

The in-use charge frequency of the entire parent data set shows a charging frequency of 1.2 charge events per vehicle-day. That is, charging occurs more often than once per day. This should weight the final results more toward chargedepleting operation. Notice, however, in both Table 3 and Table 5, the miles travelled in sustaining mode greatly outweighs the depleting mode operation. The 2001 NHTS data set utility factor for a PHEV with 30 miles depleting range is $52 \%$, in contrast to the $36 \%$ and $35 \%$ found in Tables 3 and 5 respectively.

The bias toward sustaining mode operation can be understood by looking at the distribution of distance travelled between charging events. We can make comparisons of the in-use distance between charge events to the NHTS distribution of daily vehicle miles travelled because it is assumed that NHTS vehicles are charged full before every new driving day. The fleet subset data was compared to the NHTS data to look for differences, as shown by the histogram in Figure 1.

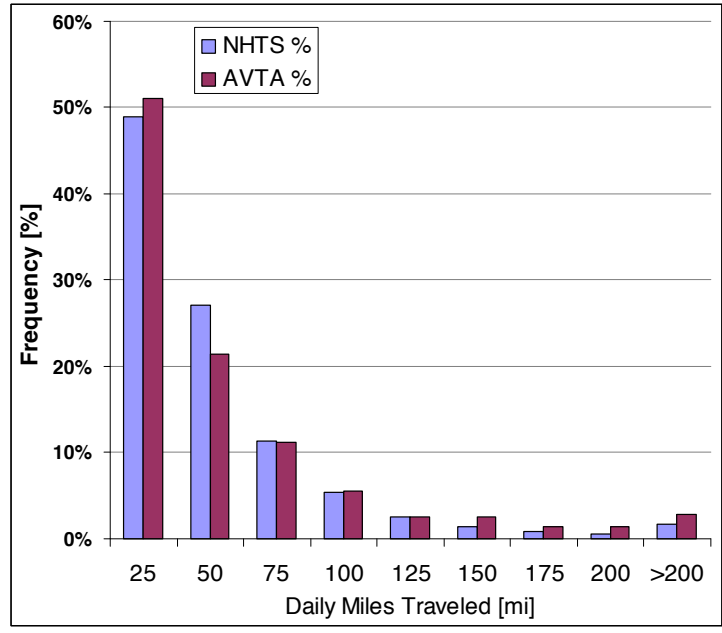

Figure 1: Histogram Comparing NHTS Daily Vehicle Miles Travelled to AVTA Fleet Distance between Charging Events

This comparison shows that the AVTA fleet drove distances greater than 125 miles per charging event more frequently than the NHTS data set. Given that the average charge-depleting range of the Hymotion Prius is approximately 30 miles. This supports the fact that the AVTA fleet drove more miles in charge-sustaining mode.

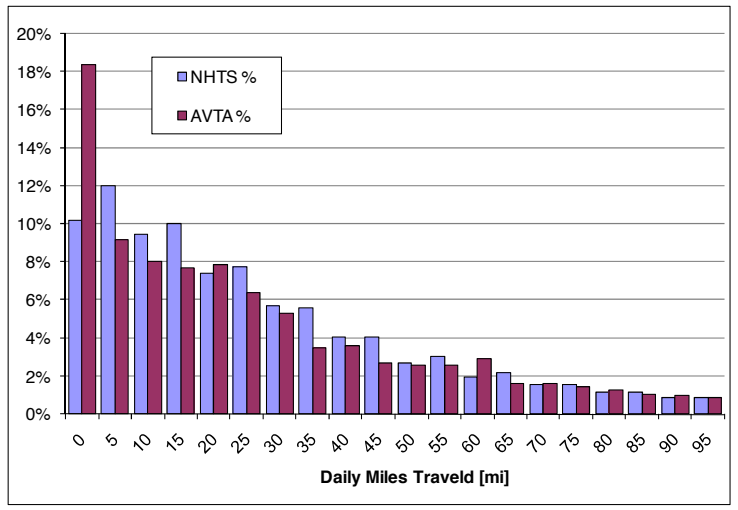

Figure 2: Higher Resolution Histogram Comparing NHTS Daily Vehicle Miles Travelled to AVTA Fleet Distance between Charging Events

Looking further into the trip distances with a histogram of higher resolution in Figure 2, it is worth noting that the data set has a large number of trips under 5 miles compared to the NHTS sample set. These short trips may have been taken within a particular campus area, or the vehicles may have been performing courier duty with many short trips.

\subsection{Driving Characteristics}

In addition to charging frequency relative to distance driven, disparity between dynamometer 
and in-use energy consumption may stem from the fact that vehicles in the fleet may have been driven in ways that are not well represented by the standard driving cycles. For example, studies have shown that vehicle energy consumption rates can be quite sensitive to driving aggressiveness (high acceleration rates and higher speeds) $[7,8]$.

\subsubsection{Modelling Vehicle Tractive Energy Output}

Average speeds or peak acceleration rates offer limited information as to how much on-board energy is required to drive the vehicle per mile. Also, high acceleration rates are independent of speeds and both contribute to higher energy consumption. It is the total road load (acceleration and drag) that make up the useful work that a vehicle has to produce. Thus, the parameter "driving intensity" is used to describe the energy per unit distance that the vehicle must provide out the drive axles.

Driving intensity is very revealing metric for comparing standard test cycles to in-use data. On the dynamometer, or with a test vehicle with axle torque sensors, output energy can be measured directly. In fleet vehicles, however, this is not feasible. Instead, a model was developed to calculate driving intensity for the in-use data based on other parameters being recorded by fleet vehicle data loggers and detailed data collected during chassis dynamometer testing.

Response Surface Modelling (RSM) techniques were applied to experimental data collected at Argonne National Laboratory's Advanced Powertrain Research Facility to generate a driving intensity correlation. This correlation linked the dynamometer recorded road load to variations in driving intensity. Input factors were speed and pedal position. The model response was tractive vehicle force. Once the modelled road load was determined, these values were integrated with the vehicle speed over time to yield total energy consumed, and finally normalized by distance to yield the intensity number. The results of the RSM are shown in Figure 3.

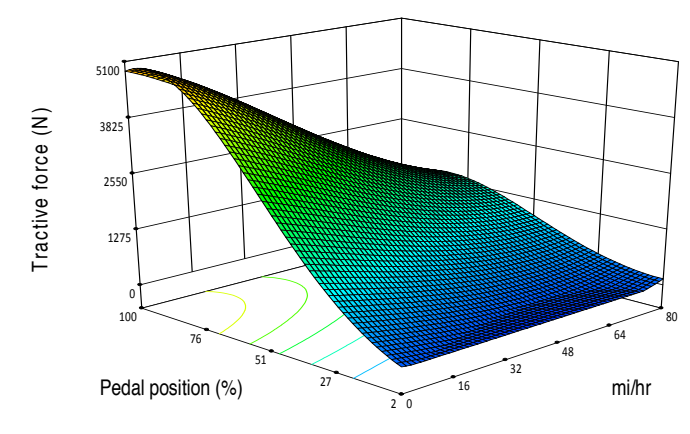

Figure 3: Response Surface Model of Tractive Force Based on Speed and Pedal Position

For this analysis, accelerator pedal position values below $2 \%$ of full pedal travel were not included, in order to analyze positive tractive effort values only. Including negative tractive effort values and integrating over the entire cycle would result in a mean driving intensity number, regardless of driving patterns. Figure 4 is the response model predicted tractive force versus the actual recorded values from the dynamometer. Sampling frequency for these data was $1 \mathrm{~Hz}$. The model reasonably predicts tractive force from these inputs, with plus and minus variations evenly distributed, mostly due to time delays between inputs and the powertrain output response.

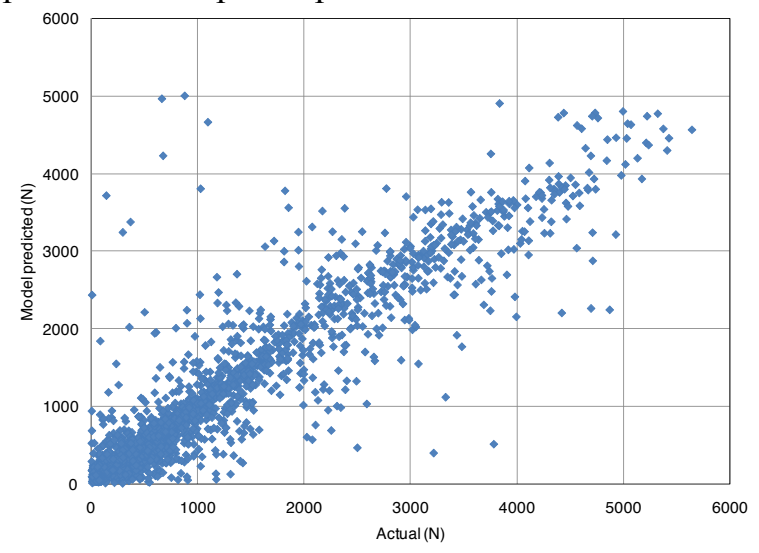

Figure 4: Response Surface Model Predicted versus Actual Road Load

Details of the model relative to the recorded load are seen in Figure 5. Only the first 200 seconds of the US06 are displayed to better show detail. From this, it may be seen that the response model accurately predicts tractive force over the cycle. Slight variations occur due to response delays in the dynamometer force calculations, calibration settings, and the lack of filtering of the response signals prior to modelling. However, integration of 
the cycle force results in reasonable predictability between the actual and model values.

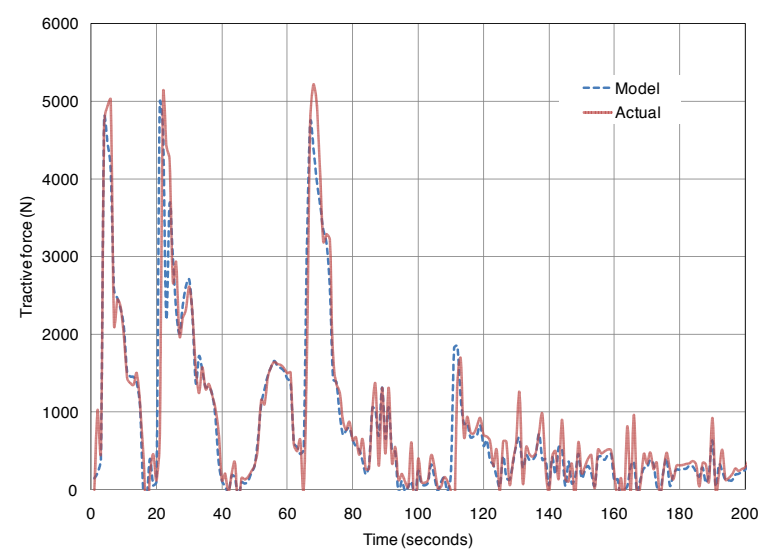

Figure 5: Tractive Force Model versus Actual Dynamometer Data, First 200 Seconds of US06 Cycle

\subsubsection{Speed and Intensity}

Driving intensity data is helpful in characterizing the driving patterns in the AVTA data set. To compare the standard drive cycles, the speeds and intensities are compared in Figure 6. The city-like drive cycles fit near the middle of the densest portion of the AVTA cluster. It appears that the densest portion driving style has a lower average speed, but higher intensity (more aggressive). The trend shows that more intense driving is more characteristic of low speed driving. Note that the HWY cycle is not representative of many trips in the data set. The HWY cycle has a high average speed but is not very aggressive.

Curiously, there are a small number of trips that are at a high average speed, but are very low in intensity. These trips are either real conditions of particularly high speed and low intensity (very steady-speed or even downhill) or possibly the input data or the intensity calculation may be in error.

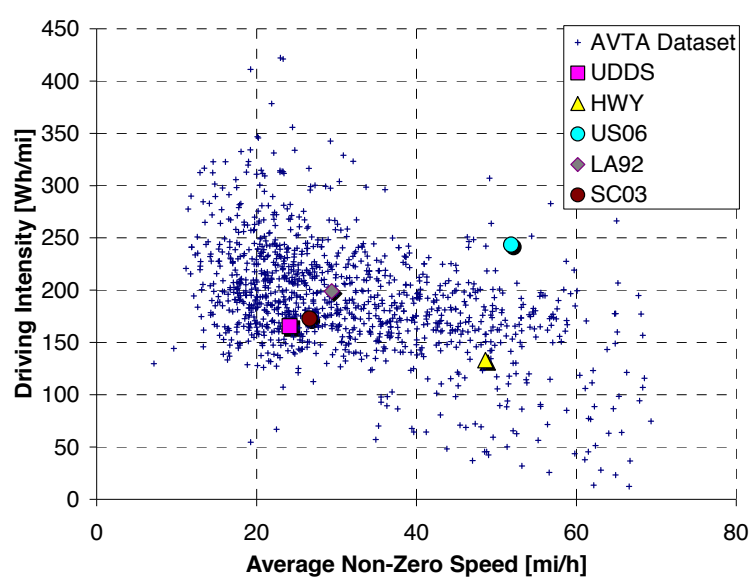

Figure 6: Average Non-zero Speed versus Driving Intensity for Fleet and Dynamometer Driving Cycles

Driving intensity estimates the useful work exerted by the powertrain during a trip. Thus, it stands to reason that no matter what mode the vehicle is in, on-board energy must be consumed at a higher rate in more intense driving. An estimation of total energy consumed during driving was made that combines the consumed battery energy and fuel. Volumetric fuel consumption was converted to energy and given a 33\% efficiency conversion (fuel lower heating value) before being added to the battery energy (DC Wh).

As seen in Figure 7, the intensity calculation of the data set loosely correlates with estimated total onboard energy consumption rate. With a few exceptions, the energy consumption points all appear above the "1:1" line indicating that there are losses between the on-board fuel used and the powertrain output. A high density cluster above the 1:1 line shows where most of the vehicle operation lies. However, there is a small portion of the data set that has very high energy consumption compared to the road load demands. (Points below the line would indicate a violation in the $1^{\text {st }}$ law of thermodynamics.) On a final observation, the intensity rate can be very low, but the energy consumption rarely falls below $200 \mathrm{Wh} / \mathrm{mi}$. 


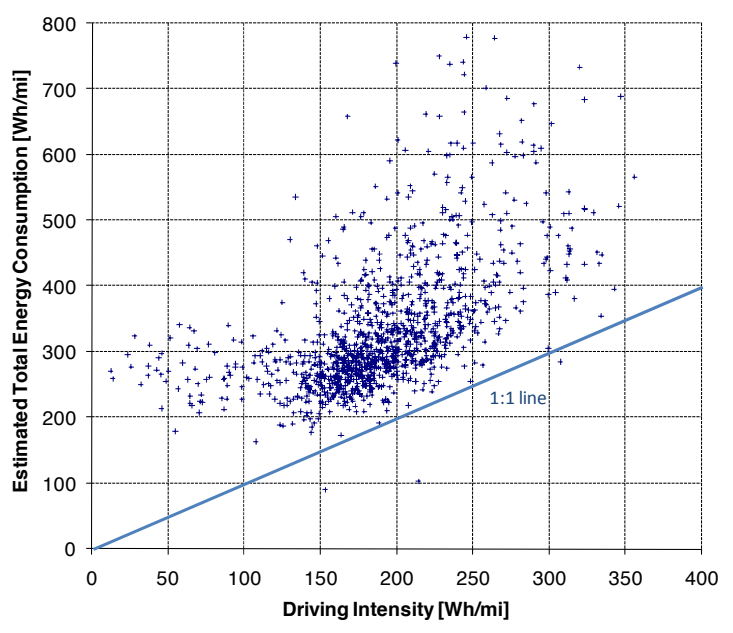

Figure 7: AVTA Fleet Driving Intensity versus Estimated Total Energy Consumption

\section{Vehicle Sensitivity to Driving Characteristics and Conditions}

When analyzing the fuel economy of a PHEV, one must not forget that two energy sources are being used and as such, fuel economy alone is no longer an appropriate singular efficiency metric. Also, the public is accustomed to seeing vehicle fuel economy in terms of miles per gallon as a relative figure of merit for energy efficiency. For example, vehicles with $40 \mathrm{MPG}$ are very efficient; $15 \mathrm{MPG}$ is very low fuel economy. PHEV fuel economy, however, is a poor relative measure of merit because MPG can range from its charge-sustaining fuel economy to infinity, depending upon the electric/fuel split. To avoid ambiguity in defining gasoline fuel use when little or no gasoline is used, fuel consumption in terms of liters per $100 \mathrm{~km}$ is a preferred metric for PHEVs.

Part of the discrepancies seen in dynamometer fuel economy compared to the on-road data set are due to the vehicle's response to different driving styles and the proportion of motive energy contributed by the battery. The design objective of the Hymotion Prius PHEV control system is to use as much electrical energy as possible. Because these vehicles are aftermarket conversions, battery contribution is limited to 20 $25 \mathrm{~kW}$ in the Toyota's charge-sustaining HEV design. In analyzing the data, the amount of fuel and battery energy consumed in the on-road data set can be identified.

\subsection{Engine-On and Fuel Consumption}

One would intuit that trips where the engine is kept off more often would result in less fuel consumption. Figure 8 shows this relationship in the fleet data set. Fuel consumption and engine state are indeed related, but there is a high amount of spread because of the many other factors determining fuel consumption.

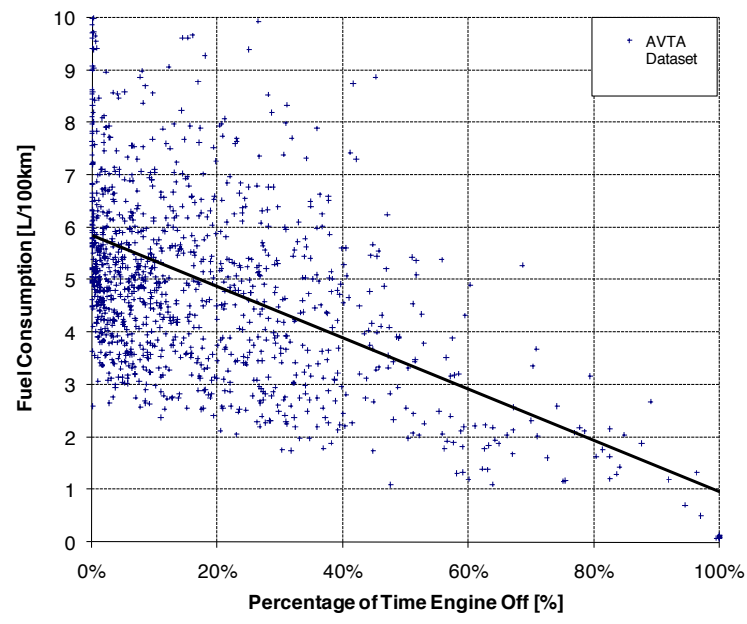

Figure 8: AVTA Fleet Engine-off Time versus Fuel Consumption

\subsection{Engine-On and Fuel-Battery Energy Split In Charge-Depleting Mode}

As shown in earlier sections of this paper, it was found that aggressiveness plays a large part in the energy consumption. However, compounding the issue is the difference in bias toward engine operation while in charge-depleting mode. There are many conditions that determine if the engine is used for propulsion. Of interest in this section is the power request from the driver, communicated through the accelerator pedal, and the vehicle speed. Trips driven only in charge-depleting mode were investigated in this section to illustrate the mix of electric and fuel use.

\subsubsection{Vehicle Speed and Battery Usage}

Speed and torque kinematics of the Prius powersplit planetary gears prevent electric-only operation at high vehicle speeds. Above $40 \mathrm{MPH}$ the engine is always being used (however, on tipout and deceleration the engine will continue to spin, but is not being fuelled). Figure 9 shows the correlation between the percentage of the powertrain energy contributed by the battery compared to the total powertrain energy using the similar calculations described in section 3.4.2. The correlation is apparent, but not very strong. 


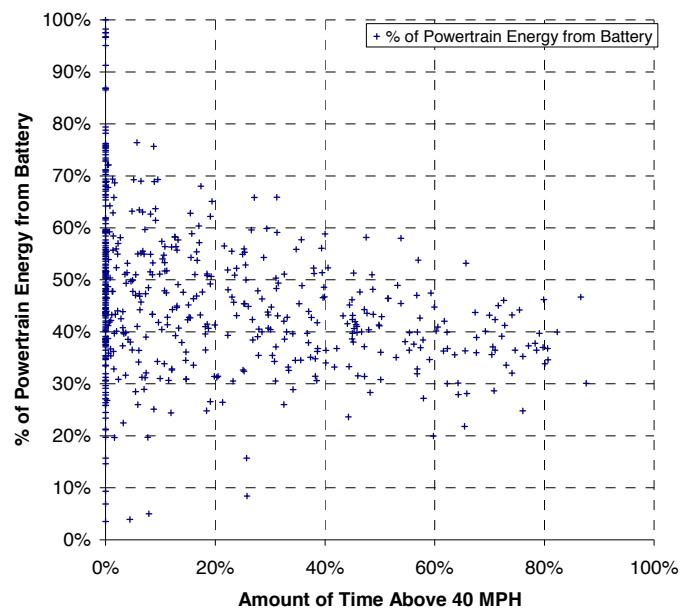

Figure 9: AVTA Fleet Time above $40 \mathrm{MPH}$ versus Percent of Powertrain Energy from the Battery

\subsubsection{Driver Demand and Battery Usage}

In defining aggressiveness, it was found that if the driver demanded more than $40 \%$ accelerator pedal, engine operation was triggered [9]. In Figure 10, the relationship between the amount of time above $40 \%$ accelerator pedal position shows a loose, but steep trend from $70 \%$ down to $30 \%$ battery contribution. The sensitivity is greater than the relationship for time above 40 MPH. It would seem that tip-in for acceleration and during driving is more important to using battery energy than is time above $40 \mathrm{MPH}$.

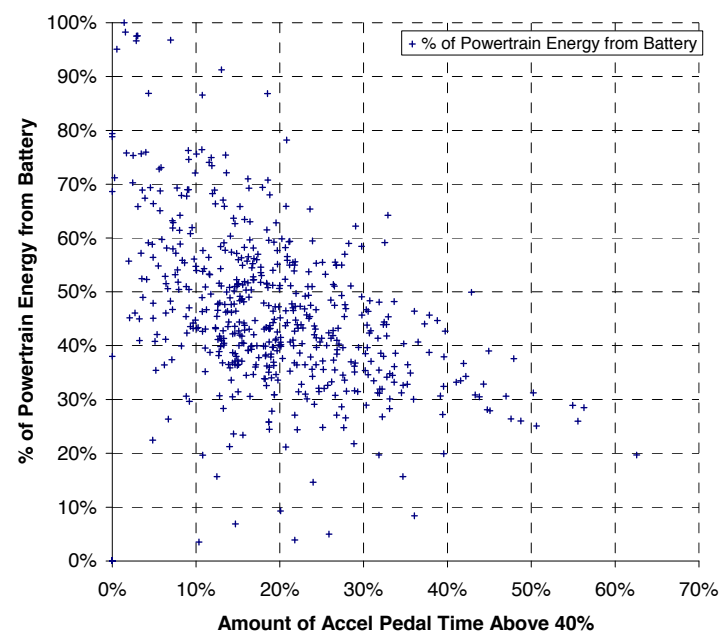

Figure 10: AVTA Fleet Time above 40\% Accelerator Pedal Position versus Percent of Powertrain Energy from the Battery

\subsection{Driver Demand and Fuel Usage}

In the end, it is the fuel usage (or reduction thereof) that is the primary objective of PHEVs. Both charge-sustaining and charge-depleting operations are analyzed for fuel consumption. Dynamometer data points representing a mix of charge-sustaining and charge depleting cycles are also analyzed. Figure 11 shows the strong trend in both the fleet and dynamometer data between fuel consumption and the percentage of time above $40 \%$ pedal position.

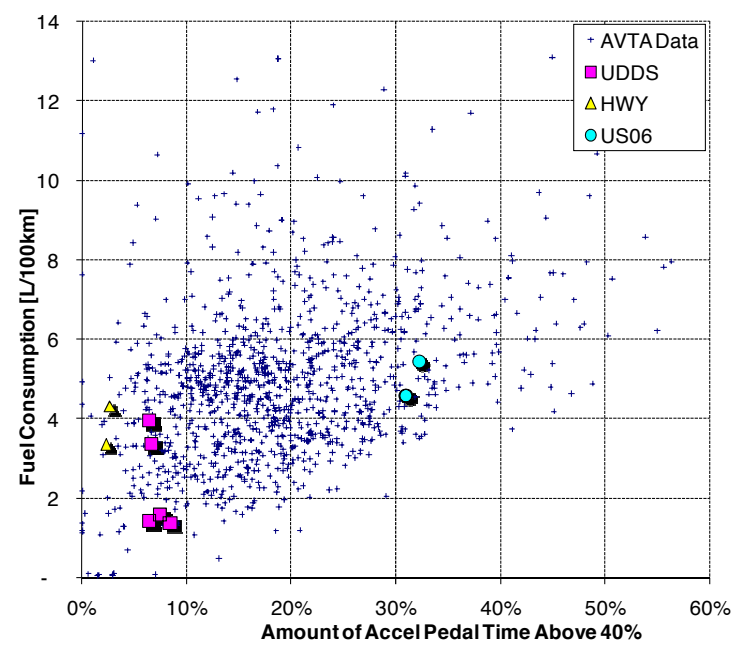

Figure 11: AVTA Fleet Time above 40\% Accelerator Pedal Position versus Fuel Consumption

Note the extremely low frequency of time above the $40 \%$ accelerator pedal position in the UDDS and HWY cycles. Looking at the data, one would guess that the UDDS and HWY are terrible predictors of the AVTA fleet operating conditions. The US06 shows a good prediction of aggregate fuel consumption, but does so with a much higher $>40 \%$ pedal position time. This data suggests that more than pure aggressiveness accounts for the fuel economy shortfall in the on-road data set.

\subsection{Vehicle Energy Consumption and Ambient Temperature}

The AVTA vehicles are deployed in locations throughout the United States and Canada and thus experience diverse climate conditions. Ambient temperature is recorded in the vehicle data loggers and thus its effect can be analyzed.

\subsubsection{Ambient Temperature and Percentage Battery Energy}

Figure 12 shows the ambient temperature and its effect on the percentage of powertrain energy coming from the battery. The points lying on the $\mathrm{x}$ - 
axis are from charge-sustaining trips. Given that the Prius has a high-voltage electric-powered $\mathrm{A} / \mathrm{C}$ system, it should be no surprise that as the temperature increases, the battery consumption mix increases.

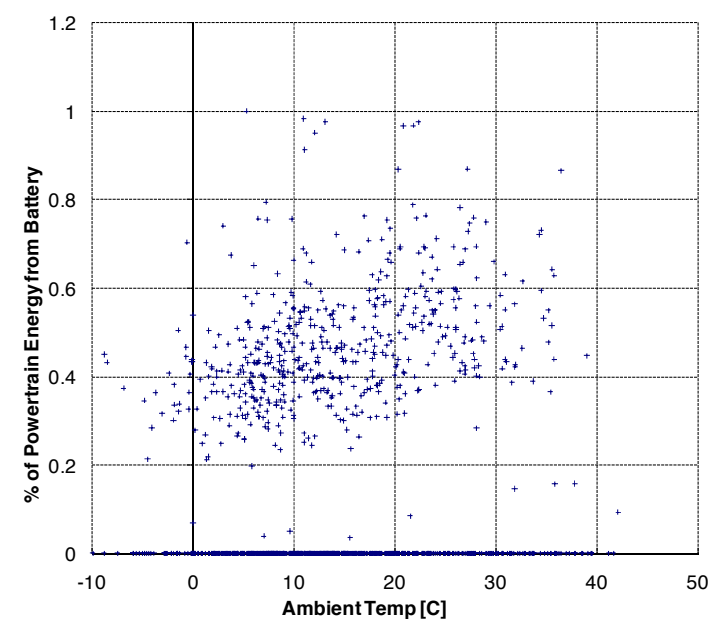

Figure 12: AVTA Fleet Ambient Temperature versus Percent of Powertrain Energy from the Battery

\subsubsection{Ambient Temperature and Total Energy Consumption}

To answer the question if ambient temperatures above and below the standard test conditions will consume more energy, the plot in Figure 13 was generated. There is a large degree of scatter, though a $2^{\text {nd }}$ order trend line indicates that the minimum total energy consumption occurs at around $23^{\circ} \mathrm{C}$.

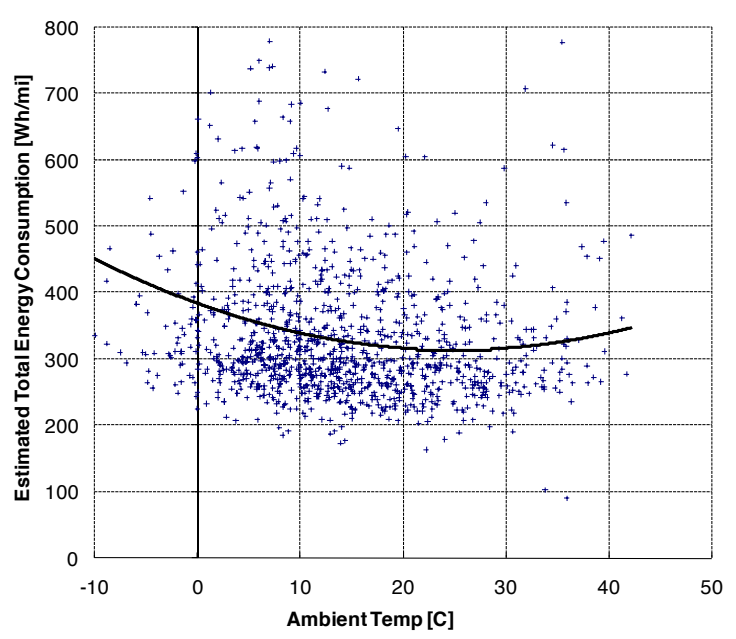

Figure 13: AVTA Fleet Ambient Temperature versus Estimated Total Energy Consumption

\subsubsection{Ambient Temperature and Fuel Consumption}

Again, fuel consumption garners the most interest, so another plot was generated to show the fuel consumption vs. ambient temperature. Figure 14 shows the same trend - a high degree of scatter and a trend minimum at roughly $25^{\circ} \mathrm{C}$.

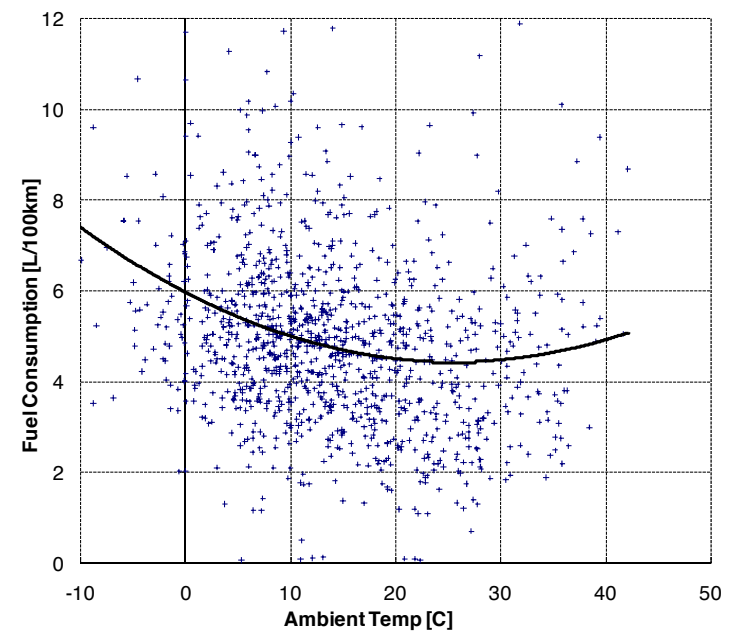

Figure 14: AVTA Fleet Ambient Temperature versus Fuel Consumption

\subsubsection{Ambient Temperature and $\mathrm{A} / \mathrm{C}$ Usage}

It has been well documented that $\mathrm{A} / \mathrm{C}$ usage can greatly increase fuel consumption [3]. In a PHEV, $\mathrm{A} / \mathrm{C}$ usage can increase both electrical energy consumption and gasoline fuel consumption, depending upon conditions. Data loggers in AVTA fleet vehicles record $\mathrm{A} / \mathrm{C}$ compressor speed over time as an indicator of $\mathrm{A} / \mathrm{C}$ usage. Trips in the 1200 trips subset were classified as having the $\mathrm{A} / \mathrm{C}$ on, based on the occurrence of $\mathrm{A} / \mathrm{C}$ compressor speed above 0 RPM. A distribution of the percent of trips with $\mathrm{A} / \mathrm{C}$ on versus ambient temperature is shown in Figure 15. There is a surprisingly high proportion of $\mathrm{A} / \mathrm{C}$ usage at all temperatures above $-10^{\circ} \mathrm{C}$. Use of the defroster, which engages the $\mathrm{A} / \mathrm{C}$ to dehumidify air blowing into the cabin, is no doubt responsible for the high proportion of $\mathrm{A} / \mathrm{C}$ usage at cold temperatures. 


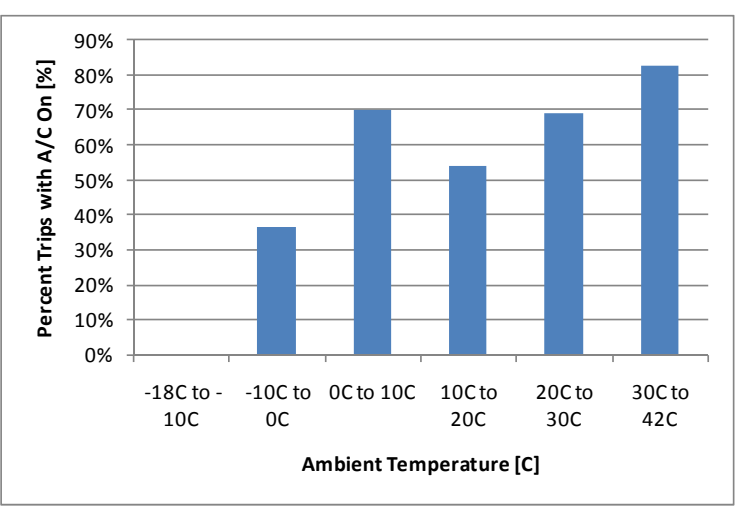

Figure 15: Distribution of A/C Compressor Usage by Ambient Temperature in the AVTA Fleet

\section{Vehicle Energy Consumption Levels}

PHEV results are conveniently shown using an $\mathrm{x}-\mathrm{y}$ plot of electrical and fuel consumption on the same graph. The full charge test given to PHEVs, which begins in charge-depleting mode and repeats cycles until the vehicle reaches chargesustaining mode, usually shows the individual cycle results on a line of constant efficiencies. If the PHEV drives a test cycle in electric-only mode, then the point appears on the zero fuel consumption axis. If the test cycle is chargebalanced, then the result appears on the zero energy consumption axis. Figure 16 describes the energy space for PHEVs. In charge-sustaining mode, driving a vehicle more aggressively, or under higher road loads, the increased fuel consumption point will appear higher on the axis. However, in charge-depleting mode, the results can take a number of directions up. Typically, a cycle with added load (either due to higher speeds or accelerations) appears on a higher constant efficiency line above the reference line. The added energy required can come solely from the battery (as in point $\mathrm{C}$ ), or from both the battery and gasoline. The latter case can result in a constant battery energy depletion rate (per mile), as is shown by point B. However, if the battery power is saturated at its highest level and the added load is a result of higher speed, the electrical depletion rate actually decreases and the result is point $\mathrm{A}$.

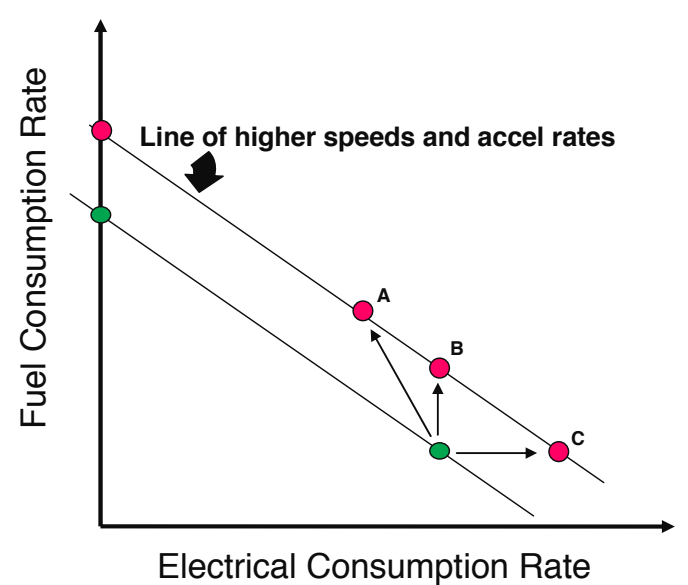

Figure 16: Method for Describing Gasoline and Electricity Consumption Space

\subsection{On-Road Data Set and Dynamometer Results}

Figure 17 shows the 1200 trip data set energy consumption space with the dynamometer drive cycle results. Note the relatively parallel CD and CS lines trading off electricity for gasoline usage indicating similar conversion efficiencies. CD points are the lower right points, the CS points at or near the x-axis. Points along the line in the middle are cycles that had both sustaining and depleting operation during the cycle (the cycle where the transition occurred). The highest energy consuming trend is from the $\mathrm{SC} 03$ test (high temperature with $\mathrm{A} / \mathrm{C}$ usage).

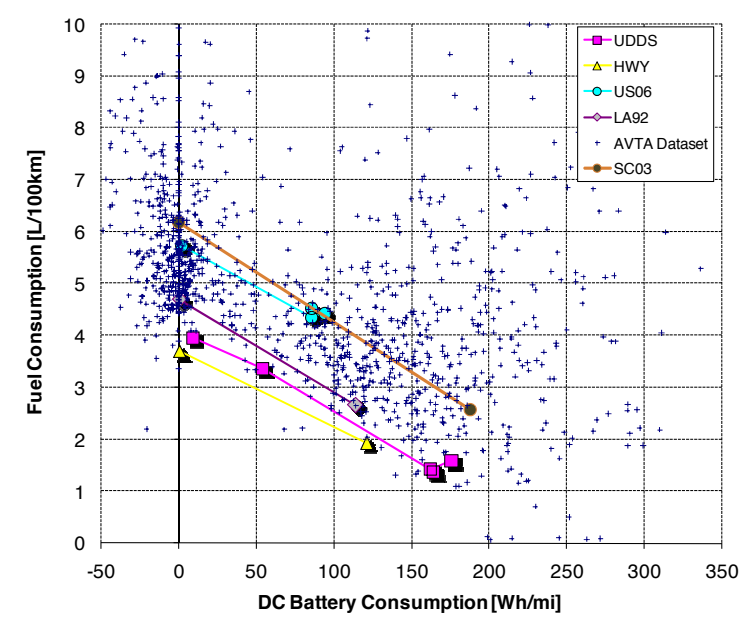

Figure 17: Energy Consumption Space for AVTA Fleet and Dynamometer Drive Cycles

Two main cluster locations dominate the energyuse space: a charge-sustaining cluster from roughly 4.5 to $6.5 \mathrm{~L} / 100 \mathrm{~km}$ (with some points higher extending up to $9.5 \mathrm{~L} / 100 \mathrm{~km}$ ), and a chargedepleting cloud from 75 to $250 \mathrm{Wh} / \mathrm{mi}$ and 1 to 5.5 
L/100km. The AVTA data shows nearly all the charge-sustaining data with higher fuel consumption than the UDDS and HWY cycles. As described earlier, aggressive driving with higher speeds, like that found in US06, corresponds to lower electric consumption rates per mile in the battery power limited Hymotion Prius. Note that the $\mathrm{SC} 03$ cycle consumes more energy because of the electrically-driven $\mathrm{A} / \mathrm{C}$ compressor usage but does so with driving speeds similar to the UDDS. Thus, the location of the charge depleting SC03 point is high on both the electric and fuel consumption axes - a location closest to the charge-depleting cloud of AVTA points mentioned above.

\section{Summary and Conclusions}

Monitoring fleet deployments of new vehicle technologies is critical to understand how performance of the technology in actual operating conditions compares to standard dynamometer testing. In the case of PHEVs, fleet demonstrations also help researchers validate assumptions used in developing vehicles and in establishing laboratory testing procedures, such as the frequency of vehicle charging and distance between charging events. Use of onboard data loggers to collect detailed information on driving style and conditions, vehicle operation, and charging patterns enables this understanding.

Many times the UDDS and HWY cycles are used as reference cycles to describe the achievements of advanced vehicles. When compared to the fleet data set analyzed in this study, they are in fact the least representative of the test cycles used, with respect to driving style, vehicle conditions, and ambient temperatures.

Using standard testing procedures and industryaccepted utility factor weighting, the on-road data set consumed $85 \%$ more fuel and $54 \%$ less electricity (per mile) than the UDDS. Consumption was $73 \%$ more on the HWY with $52 \%$ less electricity used. This disparity is partly due to a higher percentage of charge-sustaining operation in the fleet data set.

Two more aggressive cycles, the LA92, the US06, predict fuel consumption to within $2 \%$. However, they do not well represent the electrical usage (on-road consumption was 45 to $51 \%$ less).
The UF-weighted SC03 cycle, which includes the $\mathrm{A} / \mathrm{C}$-on condition found abundantly in the on-road data set, also did not predict electrical consumption rates (on-road consumption was 56\% less). Nevertheless, given the low driving intensity of this cycle and high combined gasoline and electricity consumption, as indicated above in Figure 17, this cycle demonstrates the significance of non-tractive energy demands (namely, A/C usage) on overall vehicle energy consumption.

The amount of driving in charge-depleting mode in the on-road data set was analyzed. The baseline assumption of 1 charge per driving day was close to the observed 1.2 is an encouraging find. However, the NHTS distribution of driving distances was not a good match for the on-road data, as the UF for a 30-mile charge-depleting range corresponds to a $52 \%$ expectation of chargedepleting miles, the on-road percentage of miles was only $35 \%$. More evidence of the bias toward sustaining operation is the higher frequency of very long trips (many miles beyond the depleting range). These characteristics have a fundamental impact on the amount of electrical consumption expected and the relative contribution of fuel consumption.

To further analyze the type of driving styles, the parameter "driving intensity" was established to describe the positive propulsion driving energy per distance. A satisfactory model was developed for the Hymotion Prius, using accelerator pedal position and vehicle speed to find the intensity of trips in the on-road data set. It was found that a majority of trips were at intensities higher than the UDDS, SC03, and LA92 cycles but these trips were at lower average and non-zero average speeds. The US06 and HWY cycles are at higher average non-zero speeds than a majority of the data. The US06 has an intensity that matches many trips but these trips are at much lower speeds.

It was established that the Hymotion Prius is highly sensitive to aggressive operation in the charge-depleting mode. Slight increases in accelerator pedal tip-in can prevent electric-only operation and thus, preclude fuel displacement.

Loose correlations in ambient temperature and total energy consumed on-board the vehicle and fuel consumption specifically were found. Ambient temperatures higher or lower than roughly $25^{\circ} \mathrm{C}$ were found to consume more energy. 
These finding support the inclusion of a UDDS cycle tested at $-7^{\circ} \mathrm{C}$ and the $\mathrm{SC} 03$ run at $35^{\circ} \mathrm{C}$ to better represent ambient temperature conditions and thus predict in-use energy consumption.

One perhaps surprising find in the data set is the frequency of $\mathrm{A} / \mathrm{C}$ compressor operation during driving in all but the lowest temperatures. Trips with ambient temperatures between $0^{\circ} \mathrm{C}$ and $10^{\circ} \mathrm{C}$ saw $\mathrm{A} / \mathrm{C}$ usage $70 \%$ of the time. Approximately $75 \%$ of trips at over $20^{\circ} \mathrm{C}$ saw $\mathrm{A} / \mathrm{C}$ compressor usage. Given the profound impact of $\mathrm{A} / \mathrm{C}$ compressor operation, this statistic proves to be a significant factor in the on-road data set results.

Looking at PHEV gasoline and electricity in a two-dimensional consumption space is a powerful method for making comparisons. The summary of the 1200 trip data set shows that fuel consumption in charge-sustaining mode matches a combination of the LA92 and US06. However, charge-depleting operation is not well matched by any of the drive cycles. UDDS and HWY results are virtual outliers compared to the onroad data. The SC03 is helpful in representing a consumption space location not characterized by any other cycle (high required loads at lower speeds resulting in a high fuel and electrical consumption).

In summary, it is many factors that contribute to the differences in the on-road data set from standard dynamometer testing. However, this should not discourage developers. The inclusion of additional dynamometer test cycles with varying driving conditions and the use of UF weighting are directionally correct. Also, it is important to note that trends from this study are limited to the driving and charging behavior of the fleet studied. The aftermarket conversion vehicles studied here do not necessarily manifest the performance and sensitivities of PHEVs of the future. Nevertheless, this study demonstrates the complexity of PHEV's bi-fuel operation and the importance of evaluating these vehicles across a range of conditions to accurately assess their energy consumption potential.

\section{Acknowledgments}

The authors would also like to gratefully acknowledge the sponsorship of Pat Davis, Program Manager and Lee Slezak, Manager, Advanced Vehicle Systems Simulation \&
Evaluation Team, Office of Vehicle Technologies Program, U.S, Department of Energy.

\section{References}

[1] U.S. Code of Federal Regulations, 40 CFR Part 600 -- Fuel Economy of Motor Vehicles.

[2] SAE J1711, Recommended Practice for Measuring the Exhaust Emissions and Fuel Economy of Hybrid-Electric Vehicles, SAE International, 1999.

[3] ISO 23274:2007, Hybrid-electric road vehicles -- Exhaust emissions and fuel consumption measurements -- Non-externally chargeable vehicles, International Organization for Standardization, 2007.

[4] SAE Standard J2842, Utility Factor Definitions for Plug-In Hybrid Electric Vehicles Using 2001 U.S. DOT National Household Travel Survey Data

[5] J. Smart, J. Francfort, D. Karner, M. Kirkpatrick, S. White, U.S. Department of Energy - Advanced Vehicle Testing Activity: Plug-in Hybrid Electric Vehicle Testing and Demonstration Activities, International Electric Vehicle Symposium, 2009

[6] M. Duoba, Calculating Results and Performance Parameters from Plug-In Hybrid Electric Vehicles, SAE World Congress \& Exhibition, 2009.

[7] M. Duoba, et al., Investigating Vehicle Fuel Economy Robustness of Conventional and Hybrid Electric Vehicles, International Electric Vehicle Symposium (EVS-21), 2005.

[8] R. Carlson, et al., Drive Cycle Fuel Consumption Variability of Plug-In Hybrid Electric Vehicles due to Aggressive Driving, SAE 2009-01-1335, SAE World Congress \& Exhibition, 2009.

[9] H. Iu, J. Smart, Report on the Field Performance of A123Systems' Hymotion Plug-In Conversion Module for the Toyota Prius, SAE International 2009-01-1331, 2008

[10] J. Francfort, D. Karner, R. Harkins, J. Tardiolo, Hybrid Electric Vehicle Fleet and Baseline Performance Testing, SAE International 2006-01-1267, 2005 


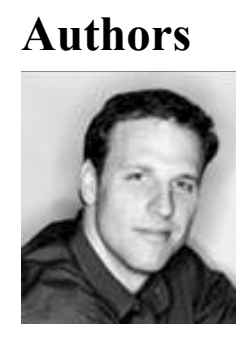

Mr. Duoba is the lead engineer for ANL's Advanced Powertrain Research Facility. He is the chair of the task force developing PHEV test procedures for SAE $\mathrm{J} 1711$ and a member of the electric vehicle test procedure task force, SAE J1634. He received his MS in Mechanical Engineering at the University of Wisconsin-Madison.

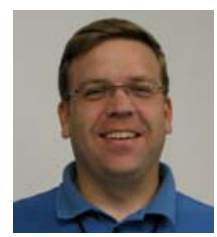

Richard "Barney" Carlson has tested and developed hybrid vehicle systems with Argonne National Laboratory for the past three years. He worked for GM R\&D in transmission development and testing for 5 years. Mr. Carlson received his BS and MS from Univ. Of California, Davis as part of the Hybrid Electric vehicle program.

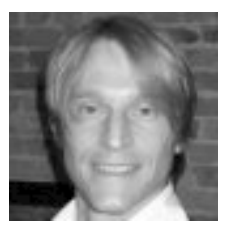

Forrest Jehlik has been at Argonne since 2005 working as a technical coordinator of the ChallengeX competition and more recently in the Advanced Powertrain Research Facility. He also worked at General Motors Research and Development/ Powertrain developing diesel combustion systems. He received a BS in Environmental Engineering at the University of California-Riverside and a MS in Mechanical Engineering at University of Wisconsin-Madison.

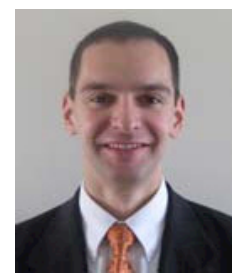

John Smart is the lead PHEV test engineer at the Idaho National Laboratory. Prior to this position, he worked at Ford Motor Company in Powertrain Product Development. Mr. Smart holds a Bachelor of Science degree in Mechanical Engineering from Brigham Young University.

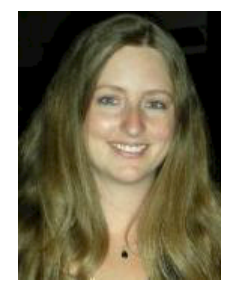

Sera White is the lead database engineer for AVTA's PHEV on-road data collection activities. Previously, she worked as a database engineer with the Geospatial Science and Engineering Group (GEOSE) at the Idaho National Laboratory. Ms. White holds a Bachelor of Science degree in Computer Science From Idaho State University. 